\title{
Difusão do gadolínio no combustível nuclear $\mathrm{UO}_{2}$
}

\section{(Diffusion of gadolinium in the $\mathrm{UO}_{2}$ nuclear fuel)}

\author{
W. B. Ferraz ${ }^{1}$, A. C. S. Sabioni ${ }^{2}$ \\ ${ }^{1}$ Centro de Desenvolvimento da Tecnologia Nuclear - CDTN/CNEN \\ C.P. 941, Belo Horizonte, MG 30123-970 \\ ferrazw@cdtn.br \\ ${ }^{2}$ Laboratório de Difusão em Materiais, Departamento de Física \\ Universidade Federal de Ouro Preto - UFOP, Ouro Preto, MG 35400-000 \\ sabioni@iceb.ufop.br
}

\begin{abstract}
Resumo
Coeficientes de difusão do gadolínio foram medidos em volume e em contornos de grão de pastilhas do combustível nuclear UO ${ }_{2}$. Entre 1505 e $1703{ }^{\circ} \mathrm{C}$, em atmosfera de hidrogênio, o coeficiente de difusão em volume pode ser descrito pela seguinte equação de Arrhenius: $\mathrm{D}\left(\mathrm{cm}^{2} / \mathrm{s}\right)=5,3 \times 10^{-3} \exp (-5,1(\mathrm{eV}) / \mathrm{kT})$. Na mesma faixa de temperaturas e na mesma atmosfera, o coeficiente de difusão do gadolínio em contornos de grão do $\mathrm{UO}_{2}$ pode ser descrito pela expressão: D' $\delta\left(\mathrm{cm}^{3} / \mathrm{s}\right)=1,1 \times 1,0-2 \exp (-6,3(\mathrm{eV}) / \mathrm{kT})$, onde D' é o coeficiente de difusão em contornos de grão e $\delta$ é a largura do contorno de grão. Nossos resultados mostram que a difusão em contornos de grão é cerca de quatro ordens de grandeza maior do que a difusão em volume nas mesmas condições experimentais. Para os diferentes isótopos do gadolínio utilizados nas experiências não foi observado efeito isotópico para a difusão do gadolínio no $\mathrm{UO}_{2}$. Não há na literatura dados sobre a difusão do gadolínio no $\mathrm{UO}_{2}$ para comparação com os resultados obtidos neste trabalho. Palavras-chave: combustível nuclear, $\mathrm{Gd}, \mathrm{UO}_{2}$, difusão em volume, difusão em contornos de grão.
\end{abstract}

\begin{abstract}
Bulk and grain-boundary diffusion coefficients were measured for gadolinium diffusion in $\mathrm{UO}_{2}$ nuclear fuel pellets. Between 1500 and $1703^{\circ} \mathrm{C}$, in hydrogen atmosphere, the bulk diffusion coefficient can be described by the following Arrhenius equation: D (cm $\left.{ }^{2} / \mathrm{s}\right)$ $=5,3 \times 10^{-3} \exp (-5,1(\mathrm{eV}) / \mathrm{kT})$. In the same temperature range and in the same atmosphere, the Gd grain-boundary coefficient in $U_{2}$ can be described as follows: $D^{\prime} \delta\left(\mathrm{cm}^{3} / \mathrm{s}\right)=1,1 x 1,0-2 \exp (-6,3(\mathrm{eV}) / \mathrm{kT})$, where $D^{\prime}$ is the grain-boundary diffusion coefficient and $\delta$ is the grain-boundary width. Our results show that the grain-boundary diffusion is ca. 4 orders of magnitude greater than the bulk diffusion in the same experimental conditions. Isotopic effect was not observed for the diffusion of the different gadolinium isotopes used in this work. There is no data in the literature about gadolinium diffusion in $\mathrm{UO}_{2}$ to compare to the present results.
\end{abstract}

Keywords: nuclear fuel, $\mathrm{Gd}, \mathrm{UO}_{2}$, bulk diffusion, grain-boundary diffusion.

\section{INTRODUÇÃO}

O combustível utilizado em reatores a água pressurizada (PWR) é o dióxido de urânio $\left(\mathrm{UO}_{2}\right)$. Entretanto, existem outras concepções modernas de combustíveis nucleares que apresentam vantagens sobre o $\mathrm{UO}_{2}$. Uma dessas alternativas ao $\mathrm{UO}_{2}$ é uma solução sólida de óxido de urânio e de óxido de gadolínio, genericamente representada por $(\mathrm{U}, \mathrm{Gd}) \mathrm{O}_{2}$, que contém até cerca de $10 \%$ em peso de $\mathrm{Gd}_{2} \mathrm{O}_{3}$ [1-5]. Nesses combustíveis, o gadolínio é utilizado como veneno queimável, ou seja, absorve nêutrons, o que permite a compensação da reatividade e o ajuste da distribuição de potência no núcleo do reator. O elemento gadolínio compõe-se de sete isótopos estáveis, cujas abundâncias são: ${ }^{152} \mathrm{Gd}(0,20 \%),{ }^{154} \mathrm{Gd}(2,18 \%),{ }^{155} \mathrm{Gd}(14,8 \%)$, ${ }^{156} \mathrm{Gd}(20,5 \%),{ }^{157} \mathrm{Gd}(15,7 \%),{ }^{158} \mathrm{Gd}(24,8 \%)$ e ${ }^{160} \mathrm{Gd}(21,9 \%)$. Sob irradiação no reator nuclear, os isótopos ${ }^{155} \mathrm{Gd}$ e ${ }^{157} \mathrm{Gd}$ absorvem nêutrons e são convertidos nos isótopos ${ }^{156} \mathrm{Gd} \mathrm{e}{ }^{158} \mathrm{Gd}$, respectivamente. A quantidade do gadolínio no combustível $\mathrm{UO}_{2}$ e a distribuição desse combustível no núcleo do reator permitem o controle da distribuição de potência no reator. Uma importante propriedade no estudo da sinterização de $\mathrm{UO}_{2}$ dopado com $\mathrm{Gd}_{2} \mathrm{O}_{3}$ e também na avaliação do desempenho do combustível no reator é a difusão do gadolínio no $\mathrm{UO}_{2}$. Entretanto, de acordo com a literatura disponível, não há medidas diretas de coeficientes de difusão do gadolínio no $\mathrm{UO}_{2}$. Este trabalho tem como objetivo implementar uma metodologia para a medida direta de coeficientes de difusão do gadolínio em volume (na rede cristalina) e em contornos de grão de cerâmicas policristalinas de $\mathrm{UO}_{2}$, utilizadas como combustíveis em reatores nucleares.

Os métodos mais precisos para o estudo experimental da difusão em sólidos são os que utilizam traçadores radioativos. Para o estudo experimental da difusão do gadolínio, entretanto, os métodos radioativos apresentam limitações. Embora seja 
possível a produção de isótopos radioativos como o ${ }^{159} \mathrm{Gd}$ e o ${ }^{161} \mathrm{Gd}$, eles não são adequados para serem utilizados como traçadores radioativos do gadolínio, porque suas meias-vidas de 18 h 56 min e 3 min 36 s, respectivamente, são curtas para estudos de difusão. Devido a isso, utilizou-se neste trabalho isótopos naturais do gadolínio como traçadores estáveis (nãoradioativos). Os perfis de difusão dos isótopos do gadolínio foram determinados por espectrometria de massa de íons secundários (secondary ion mass spectrometry - SIMS). Essa técnica, que já foi utilizada pelos autores para medir, de modo pioneiro, coeficientes de autodifusão do urânio e do oxigênio no $\mathrm{UO}_{2}[6,7]$, apresenta desempenho comparável ao de técnicas radioativas $[8,9]$.

Utilizando-se a metodologia baseada na utilização de traçadores estáveis e com análise de perfis de difusão por SIMS, foi possível medir e comparar, pela primeira vez, a difusão do gadolínio em volume (na rede cristalina) e nos contornos de grão de pastilhas do combustível nuclear $\mathrm{UO}_{2}$.

\section{PROCEDIMENTO EXPERIMENTAL}

\section{Fabricação das amostras de $\mathrm{UO}_{2}$}

Amostras de $\mathrm{UO}_{2}$ policristalinas e de alta densidade foram preparadas a partir de microesferas obtidas através do processo sol-gel. Essas microesferas, após o tratamento térmico de secagem, calcinação e redução, apresentaram diâmetros de cerca de $200 \mu \mathrm{m}$. A descrição do processo de fabricação dessas microesferas pelo processo sol-gel é feita com detalhes na referência [10]. As microesferas foram prensadas, sob uma pressão de compactação de $40 \mathrm{kN} / \mathrm{cm}^{2}$, e sinterizadas a $1700{ }^{\circ} \mathrm{C}$, durante $2 \mathrm{~h}$, sob atmosfera de $\mathrm{H}_{2}$.

A seguir, essas amostras foram re-sinterizadas a $1700^{\circ} \mathrm{C}$, durante 4 h, em hidrogênio, para estabilização do tamanho de grão.

\section{Caracterização das amostras}

A densidade das amostras sinterizadas de $\mathrm{UO}_{2}$, medida pelo método de imersão e penetração em xylol (MPI), foi de 99,3\% do valor da densidade teórica.

Para realizar a caracterização microestrutural do $\mathrm{UO}_{2}$ policristalino, as amostras foram polidas e submetidas a ataques térmicos a cerca de $1250{ }^{\circ} \mathrm{C}$, durante $60 \mathrm{~min}$, utilizando $\mathrm{N}_{2}$ nas rampas de aquecimento e resfriamento e $\mathrm{CO}_{2}$ técnico durante o patamar. A microestrutura apresentouse homogênea, praticamente sem porosidade, e o tamanho médio de grãos, medido pelo método dos interceptos, foi de $12 \mu \mathrm{m}$. Uma microestrutura típica das amostras de $\mathrm{UO}_{2}$ policristalino é mostrada na Fig. 1.

A análise química realizada por fluorescência de raios $\mathrm{X}$ mostrou que $\mathrm{o} \mathrm{UO}_{2}$ policristalino possui pureza nuclear com cerca de 220 ppm de impurezas metálicas, assim distribuídas (em ppm): Ni (12), Fe (70), Si (31), Al (15), Mg (14), Cr (43), Co $(<3)$, Mo $(<30)$.

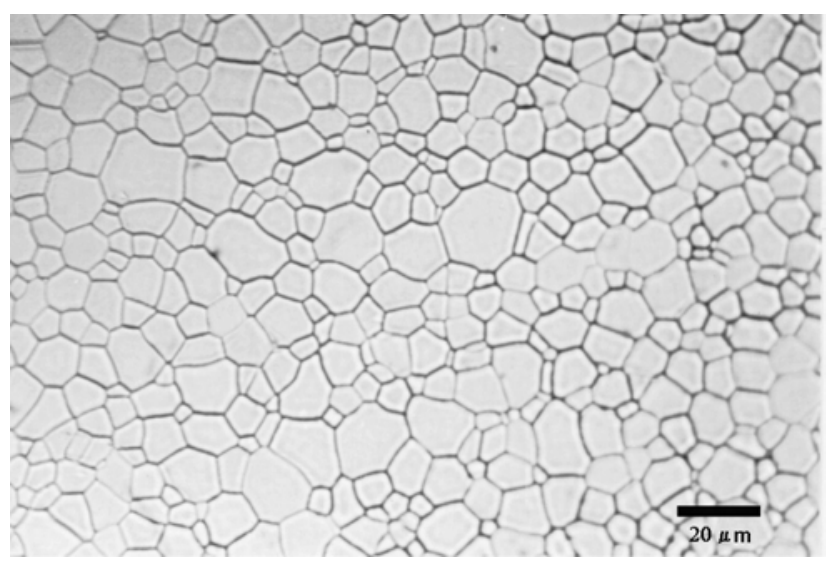

Figura 1: Microestrutura de pastilha policristalina de $\mathrm{UO}_{2}$ fabricada pelo processo sol-gel.

[Figure 1: Microstructure of a polycrystalline $\mathrm{UO}_{2}$ pellet prepared by sol-gel.]

\section{Preparação da superfície}

Para os testes de difusão, as pastilhas policristalinas de $\mathrm{UO}_{2}$ re-sinterizadas foram cortadas na forma de paralelepípedos com as dimensões $4 \times 4 \times 2 \mathrm{~mm}^{3}$. A seguir, as amostras foram polidas com pasta de diamante com granulometrias 9, 6, 3, 1 e 0,25 $\mu \mathrm{m}$, de modo a obter uma superfície polida plana, com acabamento especular.

\section{Seleção do traçador do gadolínio}

O gadolínio natural utilizado, com pureza 99,9\%, foi fornecido pela empresa Goodfellow. O gadolínio foi depositado sobre a superfície polida do $\mathrm{UO}_{2}$, por evaporação, em vácuo da ordem de $10^{-5} \mathrm{~Pa}$, na forma de um filme fino com espessura de cerca de $5 \mathrm{~nm}$.

\section{Tratamento térmico de difusão}

Os tratamentos térmicos de difusão foram realizados em um forno tubular, de resistência de Mo, na faixa de 1505 a $1703^{\circ} \mathrm{C}$, em atmosfera de gás hidrogênio puro para assegurar a estequiometria do $\mathrm{UO}_{2}$ na faixa de temperatura utilizada. A descrição detalhada da instalação de tratamento térmico utilizada é descrita na referência [6].

Em cada experiência de difusão, a amostra policristalina de $\mathrm{UO}_{2}$ foi imersa em pó de $\mathrm{UO}_{2}$ dentro de um cadinho de alumina, para proteção da amostra. Após o tratamento térmico de difusão, a superfície polida do policristal de $\mathrm{UO}_{2}$ foi limpa com acetona, em ultra-som, para a remoção de eventuais resíduos do pó de $\mathrm{UO}_{2}$

Análise dos perfis de difusão do gadolínio por Espectrometria de Massa de Íons Secundários

Neste trabalho, foram medidos por SIMS os perfis de difusão dos seguintes isótopos do gadolínio: ${ }^{155} \mathrm{Gd},{ }^{157} \mathrm{Gd}$ e 
${ }^{158} \mathrm{Gd}$. Foram medidos também, para fins de monitoramento das análises, os perfis de concentração dos isótopos ${ }^{235} \mathrm{U}$ e ${ }^{238} \mathrm{U}$ existentes naturalmente na matriz de $\mathrm{UO}_{2}$.

As análises por espectrometria de massa de íons secundários foram realizadas através de um equipamento CAMECA 4F do Laboratoire de Physique des Solides/CNRS (Meudon/Bellevue, França).

As análises SIMS dos isótopos de gadolínio e de urânio foram feitas com um feixe de íon primário de $\mathrm{O}^{+}$com energia de $10 \mathrm{keV}$. As análises foram realizadas em uma área superficial de $250 \mu \mathrm{m}$ x $250 \mu \mathrm{m}$. As intensidades dos sinais iônicos de cada isótopo analisado foram coletadas de uma região de $62 \mu \mathrm{m}$ de diâmetro no centro da área analisada.

Os perfis de concentração em profundidade foram obtidos considerando-se constante a taxa de erosão e medindo-se as profundidades das crateras por meio de um perfilômetro Tencor.

\section{RESULTADOS E DISCUSSÃO}

A Fig. 2 mostra o espectro SIMS obtido sobre uma pastilha policristalina de $\mathrm{UO}_{2}$ após a difusão a $1505{ }^{\circ} \mathrm{C}$, durante $72 \mathrm{~h}$, onde estão registrados os sinais correspondentes aos isótopos de gadolínio $\left({ }^{155} \mathrm{Gd},{ }^{157} \mathrm{Gd}\right.$ e $\left.{ }^{158} \mathrm{Gd}\right)$ e de urânio $\left({ }^{235} \mathrm{U}\right.$ e $\left.{ }^{238} \mathrm{U}\right)$. Deve ser observado que as intensidades dos sinais dos isótopos de urânio são constantes, conforme previsto. No caso dos isótopos de gadolínio, as intensidades dos sinais desses isótopos decrescem à medida que a profundidade aumenta.

$\mathrm{Na}$ medida do coeficiente de difusão do $\mathrm{Gd}$ no $\mathrm{UO}_{2}$, não foi observado efeito da massa dos diferentes isótopos

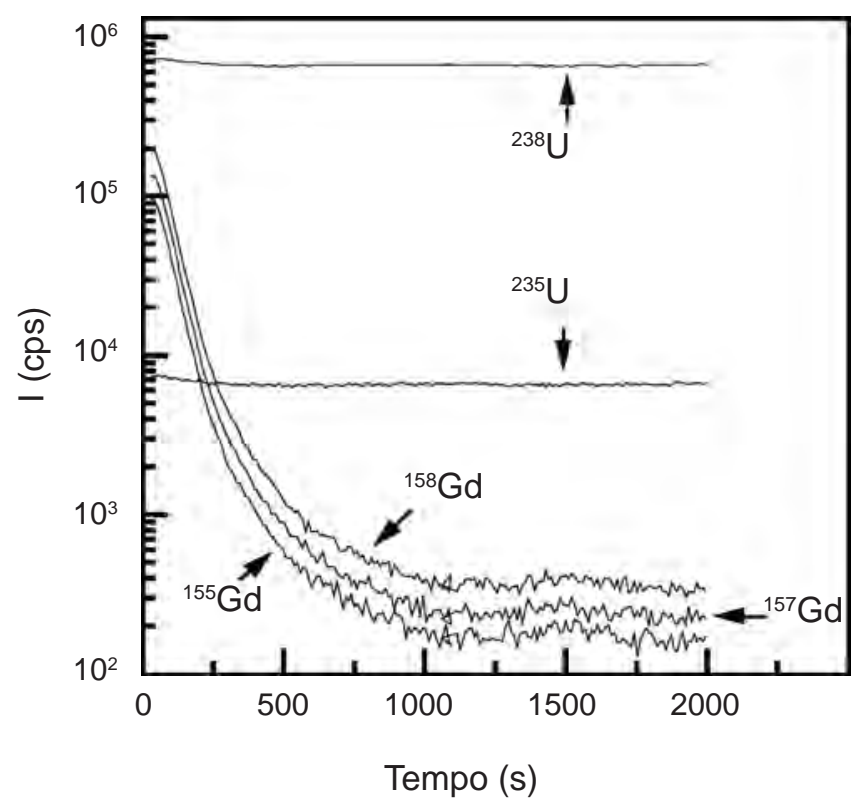

Figura 2: Espectro SIMS típico após difusão dos isótopos do gadolínio a $1505{ }^{\circ} \mathrm{C}$, durante $72 \mathrm{~h}$.

[Figure 2: Typical SIMS spectrum afetr diffusion of the $G d$ isotopes at $1505^{\circ} \mathrm{C}$ for $72 \mathrm{~h}$.] do gadolínio sobre a difusividade desse elemento no $\mathrm{UO}_{2}$. A ausência do efeito isotópico pode ser observada na Fig. 3, onde estão reproduzidos os perfis de difusão dos isótopos do gadolínio, normalizados para a concentração inicial de cada um desses isótopos, após difusão a $1505^{\circ} \mathrm{C}$, durante 72 h. Os três perfis normalizados são coincidentes, o que mostra que esses isótopos se difundem igualmente no $\mathrm{UO}_{2}$.

Portanto, neste trabalho, foi considerada para fins de cálculo apenas a difusividade de um dos isótopos de gadolínio, o ${ }^{158} \mathrm{Gd}$, simplesmente pelo fato de ser o mais abundante dos isótopos analisados. A concentração do isótopo ${ }^{158} \mathrm{Gd}$ no $\mathrm{UO}_{2}$ foi determinada através da expressão:

$$
\mathrm{C}\left({ }^{158} \mathrm{Gd}\right)=\mathrm{k} \mathrm{I}\left({ }^{158} \mathrm{Gd}\right)
$$

onde $\mathrm{C}\left({ }^{158} \mathrm{Gd}\right)$ é a concentração do isótopo ${ }^{158} \mathrm{Gd}$, em unidades arbitrárias, $k$ é uma constante de proporcionalidade e $\mathrm{I}\left({ }^{158} \mathrm{Gd}\right)$ é a intensidade dos íons de massa 158 . A descrição detalhada do cálculo de concentrações através de análises SIMS é mostrada na referência [8].

A Fig. 4 mostra um perfil de difusão do gadolínio, medido em uma amostra policristalina de $\mathrm{UO}_{2}$, após difusão a $1703{ }^{\circ} \mathrm{C}$, durante $5 \mathrm{~h}$. Nesse perfil, é possível evidenciar as contribuições das difusões em volume e em contornos de grão. A parte inicial do perfil, próximo à superfície, onde a concentração decresce rapidamente, corresponde à contribuição da difusão em volume (ou na rede).

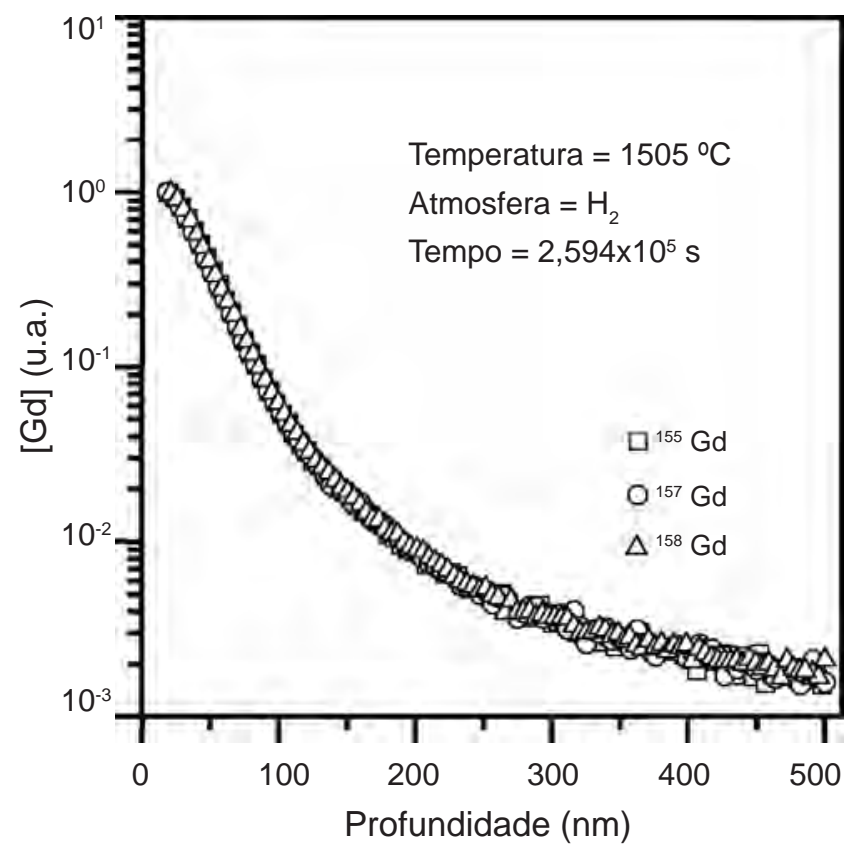

Figura 3: Curvas de penetração normalizadas dos isótopos ${ }^{155} \mathrm{Gd}$, ${ }^{157} \mathrm{Gd} \mathrm{e}{ }^{158} \mathrm{Gd}$ no $\mathrm{UO}_{2}$ policristalino.

[Figure 3: Normalized depth profile data of the ${ }^{155} \mathrm{Gd},{ }^{157} \mathrm{Gd}$ and ${ }^{158} \mathrm{Gd}$ isotopes in polycrystalline $\mathrm{UO}_{2}$.]

A parte do perfil na região mais profunda, ou seja, a 


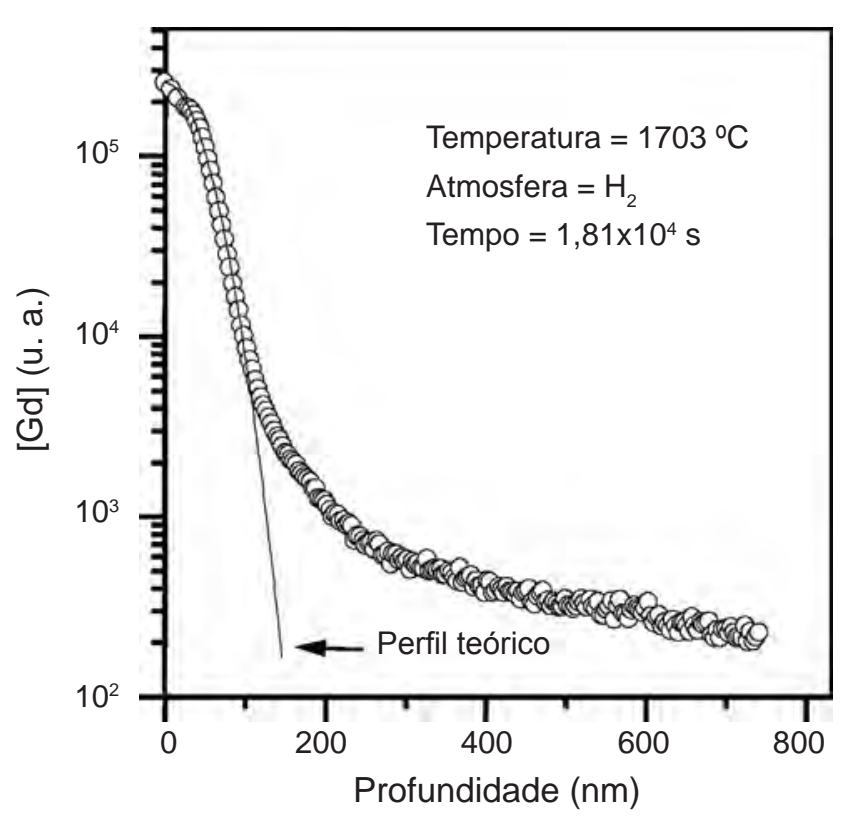

Figura 4: Curva da concentração de ${ }^{158} \mathrm{Gd}$ versus $\mathrm{X}^{2}$.

[Figure 4: Concentration of ${ }^{158} \mathrm{Gd}$ isotope as a function of $X^{2}$.]

cauda do perfil, onde a concentração decresce lentamente, corresponde à contribuição da difusão em contornos de grão. A parte intermediária do perfil, como um joelho, corresponde à superposição de contribuições das difusões em rede e em contornos de grão.

Para a determinação dos coeficientes de difusão em volume foi utilizada a solução da segunda lei de Fick para difusão em meio semi-infinito a partir de um filme fino superficial. Essa solução é dada por [11]:

$$
C(x, t)=\frac{Q}{2 \sqrt{\pi D t}} \exp \left(-\frac{x^{2}}{4 D t}\right)
$$

onde $\mathrm{Q}$ é a quantidade inicial de traçador por unidade de área, D é o coeficiente de difusão em volume, $\mathrm{C}(\mathrm{x})$ é a concentração à profundidade $\mathrm{x}$ e $\mathrm{t}$ é o tempo de difusão.

A determinação de $\mathrm{D}$ foi feita pelo ajustamento da Equação B à primeira parte do perfil de difusão, por regressão não-linear, como mostrado na Fig. 4.

Nas condições experimentais utilizadas neste trabalho, a difusão do $\mathrm{Gd}$ nos contornos de grão do $\mathrm{UO}_{2}$ foi do tipo $\mathrm{B}$, a qual satisfaz às condições de Harrison [12] dadas por:

$$
\delta<(\mathrm{Dt})^{1 / 2}<\phi / 2
$$

onde $\delta$ é largura do contorno de grão, D é o coeficiente de difusão em rede, t é o tempo de difusão e $\phi$ é o tamanho de grão.

Para a difusão intergranular no regime $\mathrm{B}$, é possível calcular o produto D' $\delta$, onde D'é o coeficiente de difusão em contornos de grão. O cálculo do produto D' $\delta$ para a difusão a partir de um filme fino, como no presente trabalho, pode ser feito pelo método de Suzuoka através da expressão [13]:

$\alpha \mathrm{D}^{\prime} \delta=2(\mathrm{D} / \mathrm{t})^{1 / 2}\left(-\partial \ln \mathrm{c} /-\partial \mathrm{x}^{6 / 5}\right)^{-5 / 3}\left(0.72 \beta^{0.008}\right)^{5 / 3}$

onde o parâmetro $\beta$ é definido por [13] :

$$
\beta=\left[(0.72)^{-1}\left(-\partial \ln \mathrm{c} / \partial \mathrm{x}^{5 / 6}\right)(\mathrm{Dt})^{3 / 5}\right]^{-1 / 0.592}
$$

A equação (4) é válida para valores de $\beta>100$ [13]. Para o cálculo do coeficiente de difusão em contornos de grão através da relação (C) foi utilizado o coeficiente de difusão em rede (D) medido neste trabalho nas mesmas condições experimentais.

A Fig. 5 mostra o perfil de difusão do ${ }^{158} \mathrm{Gd}$ no $\mathrm{UO}_{2}$ policristalino, a $1703^{\circ} \mathrm{C}$, durante $5 \mathrm{~h}$, em um gráfico de $\ln \mathrm{C}$ versus $\mathrm{X}^{6 / 5}$, que permite o cálculo do gradiente $\mathrm{d}(\ln \mathrm{C}) / \mathrm{dx}^{6 / 5}$ utilizado na Equação C.

As condições experimentais utilizadas e os resultados obtidos para a difusão do gadolínio na rede e em contornos de grãos no $\mathrm{UO}_{2}$ policristalino estão listados na Tabela I.

Nas condições experimentais utilizadas neste trabalho, o coeficiente de difusão em volume pode ser descrito pela seguinte equação de Arrhenius:

$$
D\left(\mathrm{~cm}^{2} / \mathrm{s}\right)=5,3 \times 10^{-3} \exp \left(-\frac{5.1(\mathrm{eV})}{k T}\right)
$$

Nas mesmas condições experimentais, o produto $\mathrm{D}^{\prime} \delta$, para a difusão em contornos de grão, pode ser descrito pela

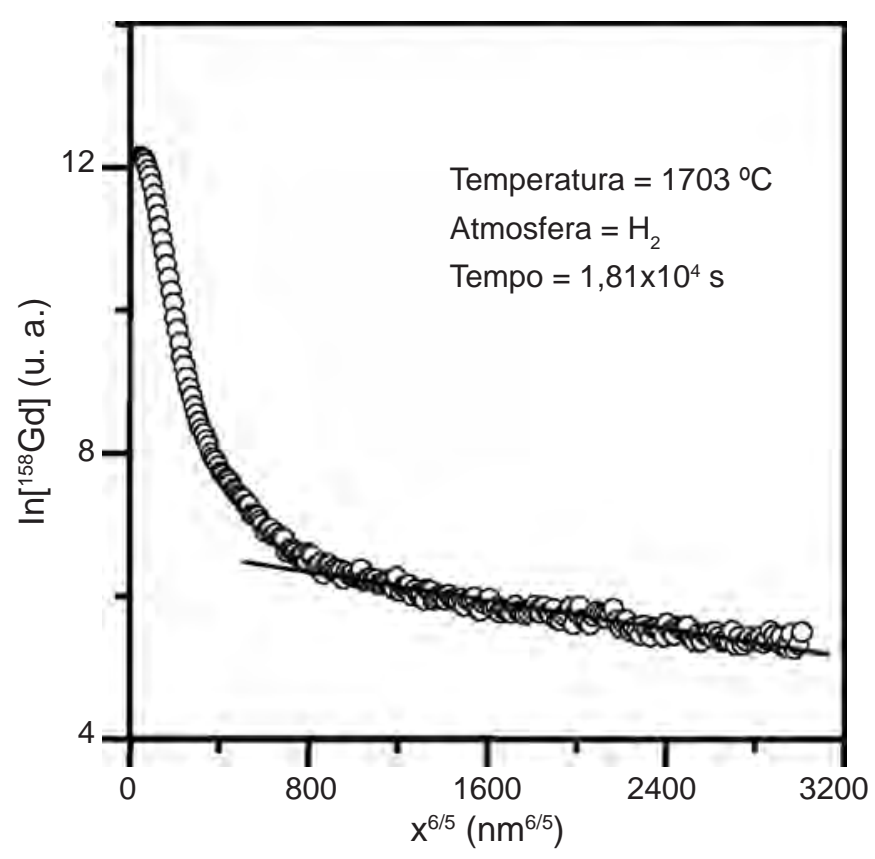

Figura 5: Curva do logarítmo da concentração de ${ }^{158} \mathrm{Gd}$ versus $\mathrm{X}^{6 / 5}$. [Figure 5: Logarithm of the concentration of ${ }^{158} \mathrm{Gd}$ as a function of $\left.X^{6 / 5}.\right]$ 
Tabela I - Condições experimentais e resultados obtidos para difusão em volume e em contornos de grãos no $\mathrm{UO}_{2}$ policristalino.

[Table I - Experimental conditions and data for volume and grain boundary diffusion in polycrystalline $\mathrm{UO}_{2}$.]

\begin{tabular}{ccccc}
\hline $\mathrm{T}\left({ }^{\circ} \mathrm{C}\right)$ & $\mathrm{t}(\mathrm{s})$ & $\mathrm{D}\left(\mathrm{cm}^{2} / \mathrm{s}\right)$ & $\mathrm{D}^{\prime}\left(\mathrm{cm}^{3} / \mathrm{s}\right)$ & $\mathrm{D}^{\prime} / \mathrm{D}$ \\
\hline 1505 & $2,594 \times 10^{5}$ & $2,03 \times 10^{-17}$ & $1,12 \times 10^{-13}$ & $5,50 \times 10^{3}$ \\
1553 & $1,514 \times 10^{5}$ & $3,52 \times 10^{-17}$ & $3,99 \times 10^{-13}$ & $1,13 \times 10^{4}$ \\
1603 & $8,640 \times 10^{4}$ & $1,51 \times 10^{-16}$ & $1,92 \times 10^{-12}$ & $1,27 \times 10^{4}$ \\
1653 & $4,320 \times 10^{4}$ & $2,81 \times 10^{-16}$ & $2,54 \times 10^{-12}$ & $9,00 \times 10^{3}$ \\
1703 & $1,810 \times 10^{4}$ & $4,56 \times 10^{-16}$ & $7,70 \times 10^{-12}$ & $1,69 \times 10^{4}$ \\
\hline
\end{tabular}

seguinte equação de Arrhenius:

$$
D^{\prime} \delta\left(\mathrm{cm}^{3} / \mathrm{s}\right)=1,1 \times 10^{-2} \exp \left(-\frac{6,3(\mathrm{eV})}{k T}\right)
$$

A energia de ativação para a difusão intergranular $\left(Q^{\prime}=\right.$ $6,3 \mathrm{eV}$ ) é maior do que a obtida para a difusão em volume $(\mathrm{Q}=5,1 \mathrm{eV})$. Teoricamente, a energia de ativação para a difusão em contornos de grão $\left(\mathrm{Q}^{\prime}\right)$ deveria ser menor do que aquela para a difusão em volume (Q). Entretanto, isso tem sido observado apenas para metais puros [13]. Para materiais não-metálicos, como os óxidos, têm sido medidos valores não só iguais a $\mathrm{Q}>\mathrm{Q}^{\prime}$, mas também $\mathrm{Q}^{\prime} \geq \mathrm{Q}[13$, 14]. Portanto, o resultado obtido neste trabalho $\left(Q^{\prime}>Q\right)$ não é surpreendente devendo estar relacionada com a segregação do gadolínio nos contornos de grão do $\mathrm{UO}_{2}$.

No diagrama de Arrhenius da Fig. 6, são comparados os coeficientes de difusão em volume (D) e em contornos de grãos $\left(\mathrm{D}^{\prime}\right)$ para a difusão do $\mathrm{Gd}$ no $\mathrm{UO}_{2}$ policristalino, determinados nas mesmas condições experimentais. Para realizar essa comparação considerou-se para $\delta$, na Equação F, o valor usual de $1 \mathrm{~nm}$ [15].

A Fig. 6 mostra que a difusão do gadolínio nos contornos de grãos do $\mathrm{UO}_{2}$ é cerca de 4 ordens de grandeza maior do que a difusão em volume, nas mesmas condições experimentais. Esse resultado está de acordo com estudo prévio da difusão do urânio no $\mathrm{UO}_{2}$ [7], e mostra que os contornos de grão são vias rápidas para a difusão de cátions no $\mathrm{UO}_{2}$.

Os resultados obtidos neste trabalho são inéditos, portanto não há na literatura dados sobre a difusão do gadolínio no $\mathrm{UO}_{2}$, que possam ser utilizados para comparação com nossos resultados.

\section{CONCLUSÕES}

Pela primeira vez foram determinados os coeficientes de difusão em volume e em contornos de grão de pastilhas do combustível nuclear $\mathrm{UO}_{2}$. Essa determinação foi realizada mediante a utilização do isótopo estável ${ }^{158} \mathrm{Gd}$ e análise dos perfis de difusão por espectrometria de massa de íons secundários (SIMS). Entre 1505 e $1703{ }^{\circ} \mathrm{C}$, em atmosfera

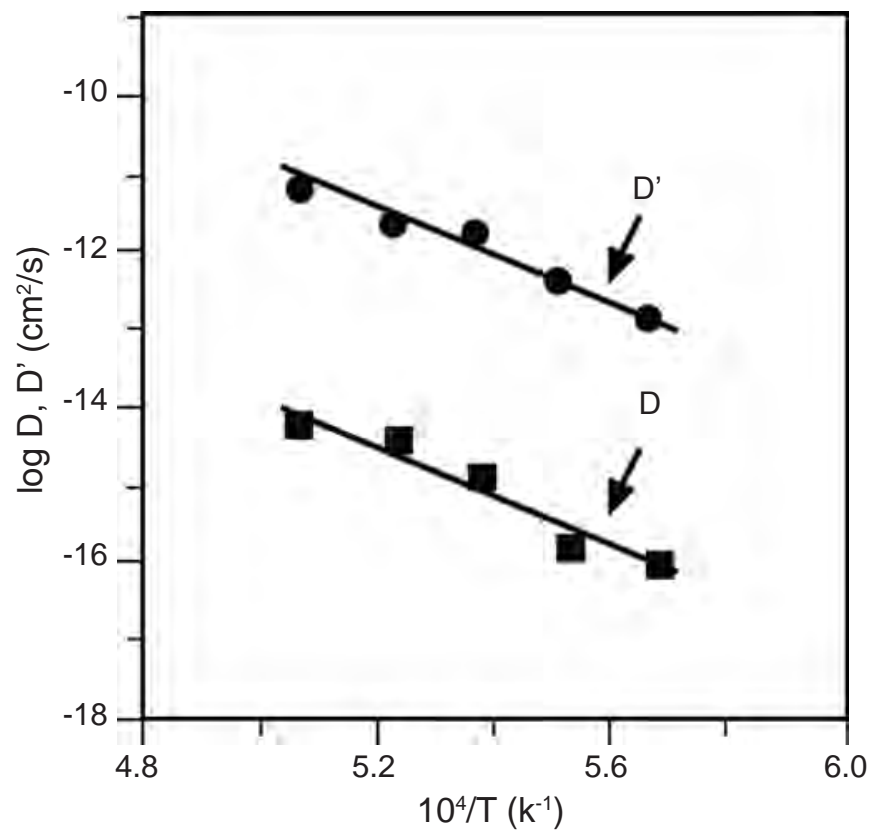

Figura 6: Diagrama de Arrhenius comparando D e D' para a difusão do Gd no $\mathrm{UO}_{2}$.

[Figure 6: Arrhenius plot of $D$ and D'for the diffudion of $G d$ in $\mathrm{UO}_{2}$.]

de hidrogênio, o coeficiente de difusão em volume pode ser descrito pela seguinte equação de Arrhenius: $\mathrm{D}\left(\mathrm{cm}^{2} / \mathrm{s}\right)=5,3$ $\mathrm{x} 10^{-3} \exp (-5,1 \mathrm{eV} / \mathrm{kT})$. Na mesma faixa de temperatura e na mesma atmosfera, o coeficiente de difusão em contornos de grãos pode ser descrito pela expressão:

$\left.\mathrm{D}^{\prime} \delta=\left(\mathrm{cm}^{3} / \mathrm{s}\right)=1,1 \times 10^{-2} \exp (-6,3 / \mathrm{kT})\right)$

Nossos resultados mostram que a difusão em contornos de grão é cerca de 4 ordens de grandeza maior do que a difusão em volume nas mesmas condições experimentais.

Para os diferentes isótopos do gadolínio utilizados nas experiências não foi observado efeito isotópico para a difusão do gadolínio no $\mathrm{UO}_{2}$.

Não há na literatura dados sobre a difusão do gadolínio no $\mathrm{UO}_{2}$ para serem comparados com os presentes resultados.

\section{AGRADECIMENTOS}

Ao CNPq e à FAPEMIG pelos auxílios concedidos, e a Christian Dolin pela realização das análises SIMS.

\section{REFERÊNCIAS}

[1] S. M. Ho, K. C. Radford, Nuclear Technology 73 (1986) 350-360.

[2] R. Yuda, K. Une, J. Nuclear Mater. 178 (1991) 195-203.

[3] T. B. Lindemer, A. L. Suttn, J. Am. Ceram. Soc. 71 ,7 (1988) 553-561.

[4] G. Gündüz, I. Uslu, J. Nuclear Mater. 231, 1-2 (1996)113-120.

[5] M. Durazzo, H. G. Riella, Advanced Powder Technology 
II 189, 1 (2001) 60-66.

[6] A. C. S. Sabioni, W. B. Ferraz, F. Millot, J. Nuclear Mater. 257, (1998) 180-184.

[7] A. C. S. Sabioni, W. B. Ferraz, F. Millot, J. Nuclear Mater. 278, (2000) 364-369.

[8] A. C. S. Sabioni, Medida de Coeficientes de Difusão a Partir de Curvas Concentração-Distância. In: Difusão em Materiais. Eds. J. Philibert, A. C. S. Sabioni, F. Dyment, Editora REM, (1996) 77-96.

[9] A. C. S. Sabioni, A. M. J. M. Daniel, W. A. A. Macedo, M. D. Martins, A. M. Huntz, F. Jomard, A. I. C. Persiano, Defect and Diffusion Forum 237-240 (2005) 277-281

[10] R. Foerthmann, G. Blass, J. Nuclear Mater. 47 (1973)
259-261.

[11] J. Philibert, Atoms Movements, Diffusion, and Mass Transport in Solids, Les Editions de Physique, Les Ulis, France (1991).

[12] L. G. Harrison, Trans. Faraday Soc. 57 (1961) 11911199.

[13] I. Kaur, W. Gust, Fundamentals of Grain Boundary and Interface Boundary Diffusion, Ziegler Press, Stuttgart, Alemanha (1988).

[13] A. C. S. Sabioni, A. M. Huntz, F. Silva, Mater. Sci. Eng. A 392 (2005) 254-261.

[14] A. Atkinson, R. I. Taylor, Phil. Mag. A 43 (1981) 979. (Rec. 20/09/2005, Ac. 09/12/2005) 